\title{
A quantitative analysis of sequential effects with numeric stimuli
}

\author{
MARK WAGNER and JOHN C. BAIRD \\ Dartmouth College, Hanover, New Hampshire 03755
}

\begin{abstract}
In a simulated absolute judgment task, subjects guessed which numeral would appear next in a random sequence. Feedback was given after every trial. The average response for the nth trial was analyzed as a joint function of both the response and the feedback for the previous trial. The results confirm those of an earlier study by Ward and Lockhead (1971). Two new models are proposed to explain sequence effects in this task of infinite difficulty. Although computer simulations based on the models fit the data reasonably well, problems remain when attempts are made to apply the same theoretical reasoning to absolute judgment tasks involving actual stimuli.
\end{abstract}

Recent research indicates that responses to stimuli in absolute identification tasks may involve two distinct components. One is the intensity of the stimulus itself; the other concerns response processes (Tanner, Rauk, \& Atkinson, 1970; Ward \& Lockhead, 1971). In an attempt to separate these components, we have explored the effects of response processes alone, in isolation from the presentation of any physical stimulus.

One class of response processes that has received attention lately is that of sequence effects (Baird, Green, \& Luce, 1980; Green, Luce, \& Smith, 1980; Staddon, King, \& Lockhead, 1980). Sequence effects exist when previous stimuli, responses, or feedback influences the current response to the current stimulus. For the method of absolute identification, Ward and Lockhead $(1970,1971)$ discussed two types of effects-assimilation and contrast. Responses assimilate toward the immediately previous response or stimulus (Trial $n-1$ ), and when the correct value of the stimulus is given by the experimenter after every trial, responses contrast with earlier response or stimulus values (Trials $n-2, n-3$, etc.).

We focus here on sequence effects in a simulated absolute identification task. It should be noted, however, that sequence effects are also found in various other psychological tasks. Ward $(1973,1979)$ and others have found such tendencies in magnitude estimation and in cross-modality matching (e.g., Baird et al., 1980; Green et al., 1980). A further by-product is reported by Cross (1973), who notes that sequence effects can lead to the underestimation of the exponents of psychophysical power functions.

One early model (Ward \& Lockhead, 1971) attempted to explain assimilation effects in the following manner. It can be shown a priori that when a

Reprint requests should be sent to Mark Wagner, Department of Psychology, Dartmouth College, Hanover, New Hampshire 03755. series of stimuli are presented, it is more probable that the intensity difference between successive stimuli will be small rather than large. For example, with 10 ordered stimuli, 10 to 1 and 1 to 10 are the only possibilities for two stimuli to be separated by nine units, whereas stimuli can be separated in 18 different ways by one unit. Ward and Lockhead account for assimilation by suggesting that subjects are aware of these probabilities and, in attempting to maximize the number of times they are correct, overestimate the frequency of small separations of successive stimuli. This would result in a large assimilation effect because successive responses would be close together. One problem with this strategy is that, by guessing fewer units of separation, the subject in no way increases the probability of guessing the correct stimulus value, since, on a specific trial, there are still 10 possible answers, each equally probable. It seems unlikely that a subject would employ a strategy that did not improve performance.

In spite of this drawback, we feel the model may still have merit. What is missing is any clear rationale for subjects to behave in this manner. In addition, the model needs increased specificity and elaboration, since, as it stands, it cannot account for contrast effects. In the last section of this paper, we provide a theoretical basis for a variant of Ward and Lockhead's original model. In addition, several new tendencies found in the numeric judgment task are discussed and incorporated within the proposed theoretical framework.

\section{METHOD}

The following experimental design is closely patterned after that of Ward and Lockhead's (1971) Study 3. The major difference is the larger number of data points collected in the present study.

\section{Subjects}

The participants were 23 Dartmouth undergraduates enrolled in an introductory psychology course. Each received nominal course credit for his/her participation. 


\section{Procedure}

Subjects were told they were participating in an extrasensory perception (ESP) experiment. They guessed which of the integers 1-9 would occur next in a sequence. Immediately following each response, a randomly selected integer was presented. From the subject's point of view, this integer represented the correct value for the previous trial; that is, the integer served as feedback. Feedback integers were presented and responses entered via a CRT terminal linked into the Dartmouth time-sharing system.

In addition to guessing which integer would occur next, the subjects also typed the value of the feedback numeral for each trial to insure that they paid attention to the feedback. Each subject participated in one session of 510 trials, with a brief rest after 255 trials.

\section{RESULTS}

Over 11,000 data points were collected, almost four times as many as Ward and Lockhead obtained. The large data base allows us to discover several patterns that previously were not apparent in the standard analyses. In addition, several new analyses can be performed, which would have been less convincing with a smaller sample size.

The average percentage of correct guesses was $11.3 \%$. (Chance performance was $11.1 \%$.) The average percentage of incorrect mimics of the feedback was $2.3 \%$, indicating that the subjects did indeed pay attention to the stimulus feedback. The average response was 5.04. This value is close to the theoretical value of 5.00 for perfectly random guessing. Contrary to Parducci's (1965) suggestion, responses were not distributed evenly. The subjects gave intermediate responses such as 4 or 5 about $1 \frac{1 / 2}{2}$ times more often than extreme values such as 1 or 2 .

Figure 1 presents the standard feedback and response analyses introduced by Holland and Lockhead (1968) to reveal sequential effects. The average response on Trial $n$ is plotted as a function of what occurred (feedback/response value) $k$ trials preceding that response. The mean response on Trial $n$ is calculated separately for pairs of feedback (left panel) or response (right panel) values on Trial $n-k$. For example, when the feedback was a 1 or a 2 on Trial $n-1$, the mean response on Trial $n$ was 4.25.

The general results agree rather well with those of Ward and Lockhead. A strong assimilation effect due to feedback exists at a lag of 1 (Trial $n-1)$ in the feedback analysis. A small, inconsistent contrast effect also occurs for lags of 2 or more. When lags are not neatly grouped in twos, as in the graph, these effects are not at all monotonic. Like Jesteadt, Luce, and Green (1977), we believe these contrast effects are relatively small and perhaps of little consequence. Unlike these authors, however, we do not think that contrast effects are an artifact of the mode of analysis. (They occur robustly in another type of analysis to be presented later.) Also, they are not an artifact of the assimilation effects found for Trial $n-1$ but,

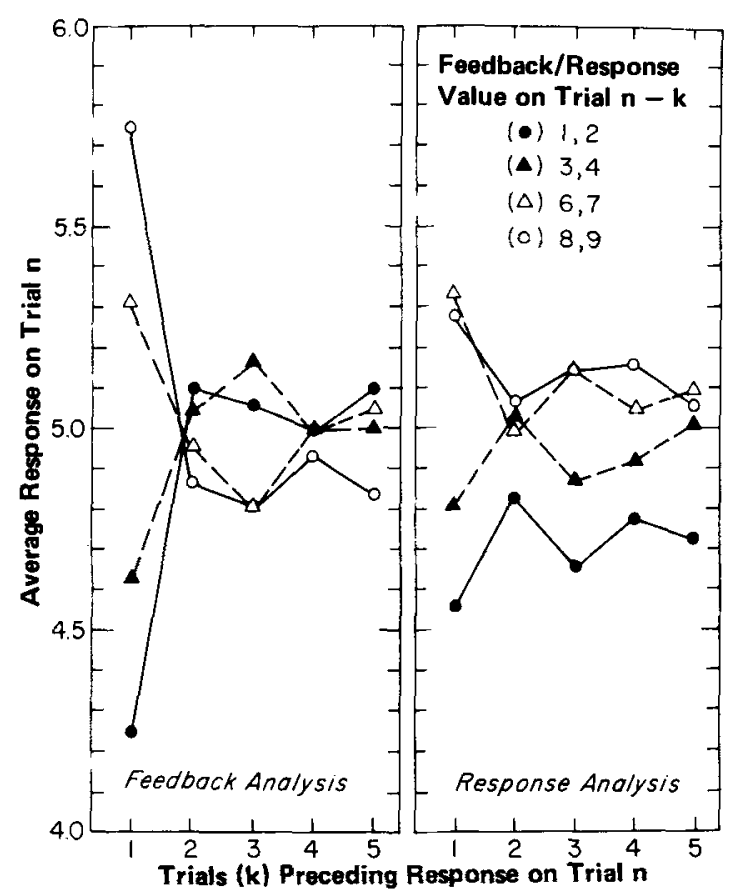

Figure 1. Average response on Trial n following a given feedback/response on Trial $n-k$.

rather, appear to be caused by an actual suppressive mechanism.

Unlike the results of Ward and Lockhead, ours show an assimilation effect due to prior responses (right panel in Figure 1). These response effects are smaller than those due to feedback. No contrast effect is evident.

In both the feedback and response analyses, the clearest results are those for a lag of 1 . The average value for the response on Trial $n-1$ may be viewed as a joint function of both the previous responses and the feedback. The largest assimilation effects occur when the feedback and the response for Trial $n-1$ are nearly the same. When the feedback and the response on Trial $n-1$ are far apart, the response on Trial $\mathrm{n}$ is close to the mean of 5.0. Furthermore, when the feedback and the response for Trial $n-1$ are approximately equal, subjects tend to give responses that are nearly the same as their last response. When the feedback and the response for Trial $n-1$ are highly disparate, subjects tend to give responses that are relatively more distant from their last response.

Assimilation effects imply that subjects use numbers on Trial $n$ that are close to the value of the feedback or the response on Trial $n-1$. To examine this assertion directly, an analysis was performed on triplets of feedback and responses of the form $[R(n-1)$, $S(n-1), R(n)]$. In this case, the response on Trial $n$, $R(n)$, is subtracted from the feedback on Trial $n-1$, 


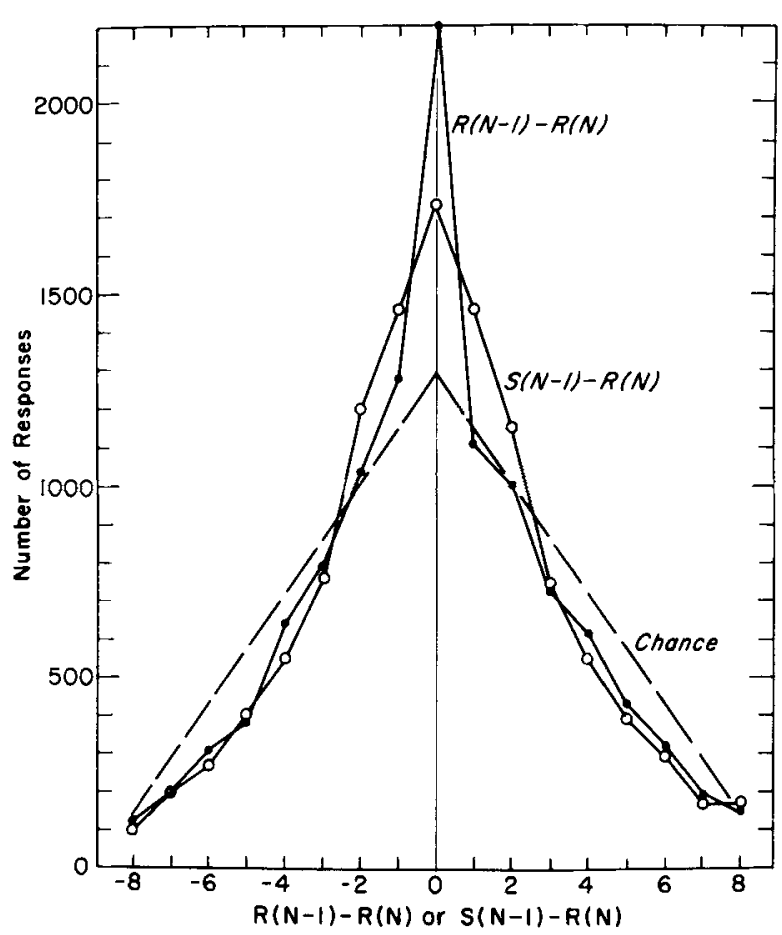

Figure 2. Frequency distribution of the differences between the feedback/response on Trial $n-1$ and the response of Trial $n$. The dashed line represents the expected number of responses by chance alone for each difference.

$S(n-1)$. The number of trials satisfying each difference score (D) is then cumulated into bins. A similar cumulation is made for all values of $R(n-1)-$ $R(n)$. In Figure 2, the frequency polygons resulting from these calculations are compared with the results expected by chance alone.

Two observations can be made about these data. First, for $R(n-1)-R(n)$, only one point differs greatly from chance-when $R(n-1)-R(n)$ is equal to zero (when subjects repeat the old response value). This particular difference is highly significant by a normal approximation to the binomial $(p<.001)$. The tendency for response repetition was noted earlier (Garner, 1953; Tanner et al., 1970) in similar tasks. On the other hand, the feedback effect $[S(n-1)-$ $\mathbf{R}(\mathrm{n})]$ is more graded. Subjects tend to respond within two units of the feedback value.

In order to determine whether an interaction is present between the feedback and response effects, a normalization procedure is used. Instead of one unit's being added to the cumulation for each triplet satisfying the condition $R(n-1)-R(n)=D$, another number, $M=C(D) / T(D)$, is added to the cumulation. Here, C(D) is the number of triplets that should satisfy each difference score, $D$, by chance alone. $T(D)$ is the total number of triplets actually satisfying the difference score. We would expect the cumulation to have the value $M \times T(D)=[C(D) / T(D)]$ $\times T(D)=C(D)$. In other words, the cumulation for
$R(n-1)-R(n)$ is reduced to chance. Now a new cumulation is made for each difference score $S(n-1)$ $-R(n)=D$, using the same values of $M$ obtained earlier for each triplet. If the feedback distribution is at all dependent upon the response distribution, we would expect that the elimination of the response effect should somehow alter the shape of the feedback distribution. In fact, the $S(n-1)-R(n)$ polygon resulting from this normalization procedure is almost exactly the same as that shown in Figure 2. A similar normalization upon $S(n-1)-R(n)$ yields no differences in the $R(n-1)-R(n)$ polygon. These analyses strongly support the notion that the feedback and response processes are orthogonal.

Cumulations similar to those found in Figure 2 were made for Trials $n-2, n-3, n-4$, and $n-5$. Once again, the response on Trial $n$ was subtracted from the feedback and response values for each of these trials. The response repetition effects found for Trial $n-1$ are apparent for these trials, and all the results are significantly different from chance $(p<$ $.001)$. The response repetition effects do not appear to decrease in strength even after five trials.

The feedback effects are a bit more complex. Whereas on Trial $n-1$ more feedback values are repeated than would be expected by chance, fewer feedback values are repeated than would be expected by chance for Trial $n-2(p<.001)$. In other words, feedback repetition was suppressed. This suppression also occurred, albeit in a weaker form, for Trial $n-3$ $(p<.01)$. Repetitions did not differ significantly from chance for Trials $n-4$ or $n-5$.

\section{THEORETICAL MODELS}

We propose two very different models for sequence effects in this numeric task of infinite difficulty. The first model treats sequence effects as the product of a learning process. The second model supposes that they are the result of a person's subjective concept of randomness.

The learning model entails the following scenario: On a given trial, the subject guesses an integer, and the experimenter provides feedback. Knowing that extrasensory perception is not precise, the subject compares the response with the experimenter's feedback and determines the error. This error provides a correction factor to be applied to the response, yielding a new response available for use on the next trial. However, we assume the correction factor will be only a fraction of the error itself. Thus, the new response will be assimilated only a portion of the distance between the original response and feedback values. This means that when assimilation occurs, responses will fall at intermediate points, not at the ends of the response continuum. We also assume that the corrected response, while available for use on the next trial, will be selected on only a fraction of the 
trials; otherwise, the subject selects an integer at random. In short, according to this model, the subject attempts to learn the correct response for the next trial. Just as in probabilistic learning theory, the largest adjustment of a response will occur on trials when the subjects are most in error (Estes, 1964).

A representation of this model requires two parameters. The first parameter, $\alpha$, determines the portion of the distance between $R(n-1)$ and $S(n-1)$ that the response on Trial $n$ will move toward the feedback. The second parameter, $\beta$, determines the probability with which the subject guesses randomly on the next trial. In other words, $\beta$ acts as a noise factor.

Mathematically, this may be expressed in the linear form:

$$
R(n)=R(n-1)+\alpha[S(n-1)-R(n-1)], \chi \geqslant \beta ;
$$

$\mathrm{R}(\mathrm{n})$ is a randomly chosen integer (1-9), $\chi<\beta$.

Here $\chi$ represents a randomly chosen real number between 0 and 1.

The standard feedback and response analyses, such as those displayed in Figure 1, were conducted with the output of a computer simulation of this model $(10,000$ trials). The results of these analyses are displayed in Figure 3. Assimilation effects are mimicked reasonably well by the simulation $(\alpha=.66$, $\beta=.66)$.

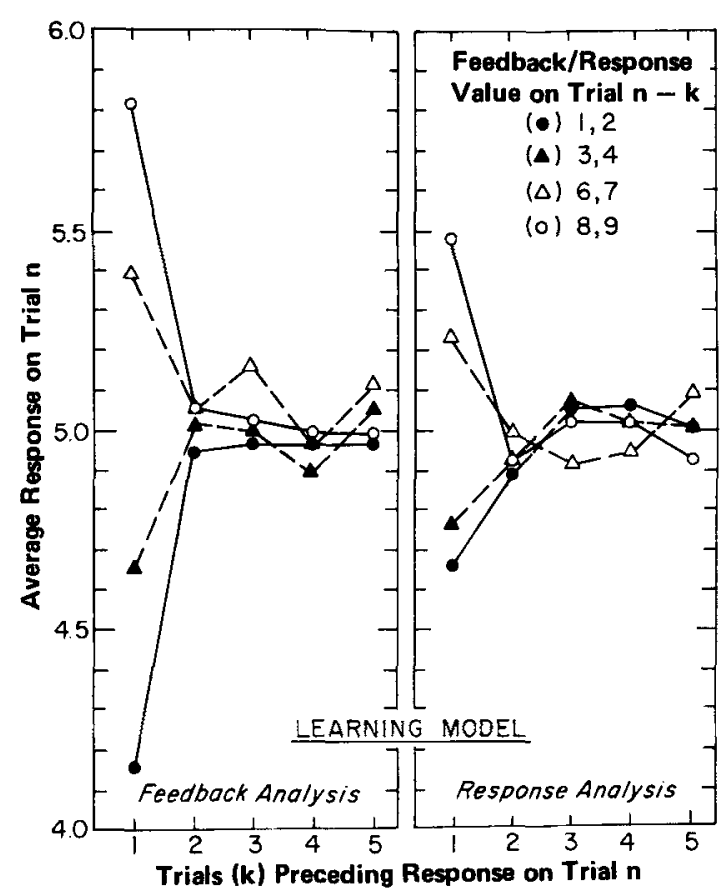

Figure 3. Average response on Trial n following a given feedback/response on Trial $n-k$. The data were generated by computer simulation of the learning model. (See text for details.)
In addition, the results at a lag of 1 were analyzed in the same manner as the original data. A computer simulation of this model was compared with the original mean $R(n)$ data for the triplet $[R(n-1)$, $\mathrm{S}(\mathrm{n}-1), \mathrm{R}(\mathrm{n})]$. A $\chi^{2}$ analysis revealed virtually no difference between the two sets of data $\left[\chi^{2}(64)<35.5\right]$. Additionally, the cumulative polynomial for $S(n-1)-$ $R(n)$ is graded as in the empirical data.

The major advantage of this model is one of theoretical clarification. It gives a psychological rationale for the existence of sequence effects beyond the mere statement of equations. This would be especially true when physical stimuli are presented. If our analogy to learning theory is correct, much of the extensive mathematics of probabilistic learning theory may be applicable to sequence effects. There are problems with this model, however. Most importantly, like most linear models, it cannot account for contrast effects ${ }^{1}$ because the amount of response change is proportional to the difference between the feedback and the response on Trial $n-1$.

The second model, called the repetition model, assumes that sequential effects result from the incorrect beliefs that subjects possess about the concept of randomness. Reichenbach (1949) was the first to show that subjects cannot produce a random sequence even when specifically instructed to do so. Since that time, at least 15 studies have documented this effect. (See Wagenaar, 1972, for a review.) The basic findings are that subjects tend to make responses that are greatly separated, and they tend not to repeat themselves. This is the famous "gambler's fallacy," often known as the "law of averages." Subjects believe that repeats in a random sequence are unlikely, while numbers that have not occurred for a while are more probable. Thus, if subjects were responding according to their notion of randomness, without regard to feedback, we would expect few repetitions. (Note that these findings directly contradict Ward \& Lockhead's, 1971, assumptions about the perception of random sequences.)

In fact, feedback does influence the subject's response. According to the repetition model, it does so because people do not believe that the sequence to which they are responding is entirely random. During debriefing, many of our subjects reported that they were actively searching for patterns, but the only pattern consistently reported was that feedback values were repeated too often. A similar effect has been reported in the literature on perceived randomness (Mittenecker, 1953; Wagenaar, 1970; Zwaan, 1964): Subjects report that sequences are random when they contain fewer repeats than would normally occur by chance. In the repetition model, we propose that subjects overadjust their responses to compensate for the one pattern that they do see (feedback diads). That is, they repeat the previous feedback value. 
Otherwise, subjects act in accordance with the "law of averages." Subjective reports during debriefing support this proposition. Subjects reported employing the strategy of responding repeatedly with a number that hadn't occurred for a period of time, producing a large number of response repetitions. Conversely, subjects avoided feedback values that had occurred recently (i.e., on Trials $n-2, n-3$ ), thus creating the feedback suppression effects.

Mathematically, the repetition model contains three parameters, and may be expressed as a series of steps.

(1) Randomly select an integer I from 1 to 9.

(2) Randomly select an $x$ value $(0<x \leqslant 1)$.

(3) Depending on $x$, go to case a, b, c, or d: (a) $I=$ $S(n-1)$, then $R(n)=I(x \leqslant \alpha)$. (b) $I=R(n-1)$, then $R(n)=I(\alpha<x \leqslant \beta)$. (c) If $I=S(n-2)$ or $S(n-3)$, return to Step $2(\beta<x \leqslant x)$. (d) $R(n)=I(x<x \leqslant 1)$. Step $3 c$ is responsible for stimulus suppression and is appropriate for the two trials $(n-2$ and $n-3)$ that were significant in the empirical results.

The results of a computer simulation of this model $(8,000$ trials) were treated via the standard analyses (Figure 1); approximately equal weights were given to steps $a, b$, and $c$. The results of the simulation are displayed in Figure 4, in which both assimilation and contrast effects are apparent.

The computer simulation of this model was compared with the original mean $R(n)$ data for the triplet $[R(n-1), S(n-1), R(n)]$. A chi-square analysis re-

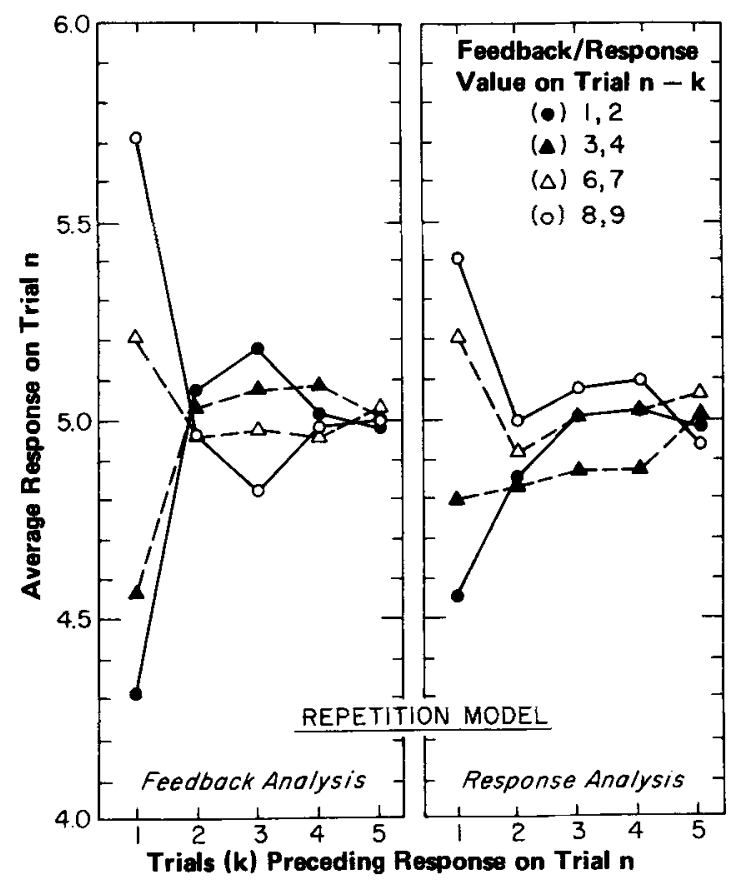

Figure 4. Average response on Trial n following a given feedback/response on Trial $n-k$. The data were generated by computer simulation of the repetition model. (See text for details.) vealed that the fit of the simulation to the empirical findings is excellent $\left[\chi^{2}(64)<35.5\right]$.

Despite this success, there are several drawbacks of the repetition model in its present form. First, the cumulative polygon for $S(n-1)-R(n)$ does not have the graded appearance of the empirical data (Figure 2). A more complete model would have to account for that. Second, the response analysis is not perfectly reproduced. The probability of repeating $R(n-k)$ decreases in an approximately geometric fashion as a function of trials, $k$. [As in a Markov chain model, the probability that a string of repetitions will last $\mathbf{n}$ trials is $\alpha$ to the nth power, where $\alpha$ is the probability of of a single repetition. However, the probability of repeating $R(n-k)$ is only approximately geometric since there is a small, but existent, chance that $R(n-k)$ may be given, even if not part of a string of repetitions.] A more satisfactory model would account for the durability of the response repetition effects. Third, it may be asked whether subjects in an absolute judgment task (with actual stimuli) look for patterns in the sequence of stimuli, as we have suggested for numeric stimuli. Finally, of course, both the learning and repetition models are incomplete in one critical way. In a task in which actual stimuli are presented, one does not expect the subject to choose responses randomly from the available set (except on early trials). The region in which responses will fall is largely determined by the actual value of the stimulus. Any complete model of sequential effects must take that basic fact into account. Nonetheless, the learning and repetition models alert us to some potentially relevant response variables in standard psychophysical tasks.

\section{REFERENCES}

Baird, J. C., Green, D. M., \& Luce, D. Variability and sequential effects in cross-modality matching of area and loudness. Journal of Experimental Psychology: Human Perception and Performance, 1980, 6, 277-289.

Cross, D. V. Sequential dependencies and regression in psychophysical judgments. Perception \& Psychophysics, 1973, 14, 547-552.

Estes, W. K. Probability learning. In A. W. Melton (Ed.), Categories of human learning. New York: Academic Press, 1964.

GarNer, W. R. An informational analysis of absolute judgments of loudness. Journal of Experimental Psychology, 1953, 46, 373-380.

Green, D. M., Luce, R. D., \& Smith, A. F. Individual magnitude estimates for various distributions of signal intensity. Perception \& Psychophysics, 1980, 27, 483-488.

Holland, M. K., \& Lockhead, G. R. Sequential effects in absolute judgments of loudness. Perception \& Psychophysics, $1968,3,409-414$.

Jesteadt, W., Luce, R. D., \& Green, D. M. Sequential effects in judgments of loudness. Journal of Experimental Psychology: Human Perception and Performance, 1977, 3, 92-104.

Mittenecker, E. Perseveration and persönlichkeit. Zeitschrift für experimentelle und angewandte Psychologie, 1953, 1, 5-31.

PARduccl, A. Category judgment: A range-frequency model. Psychological Review, 1965, 72, 407-418. 
Reichenbach, H. The theory of probability. Berkeley: University of California Press, 1949.

Staddon, J. E. R., King, M., \& Lockhead, G. R. On sequential effects in absolyte judgment experiments. Journal of Experimental Psychology: Human Perception and Performance, 1980, 6, 290-301.

Tanner, T. A., Jr., Rauk, J. A., \& Atrinson, R. C. Signal recognition as influenced by information feedback. Journal of Mathematical Psychology, 1970, 7, 259-274.

WagenaAr, W. A. Appreciation of conditional probabilities in binary sequences. Acta Psychologica, 1970, 34, 348-356.

WagenaAR, W. A. Generation of random sequences by human subjects: A critical survey of literature. Psychological Bulletin, 1972, 77, 65-72.

WARD, L. M. Repeated magnitude estimates with a variable standard: Sequential effects and other properties. Perception \& Psychophysics, 1973, 13, 193-200.

WARD, L. M. Stimulus information and sequential dependencies in magnitude estimation and cross-modality matching. Journal of Experimental Psychology: Human Perception and Performance, 1979, 5, 444-459.

WArD, L. M., \& Lockhead, G. R. Sequential effects and memory in category judgments. Journal of Experimental Psychology, $1970,84,27-34$.

WARD, L. M., \& Lockhe AD, G. R. Response processes in absolute judgment. Perception \& Psychophysics, 1971, 9, 73-78.

ZWAAN, E. J. De afhankelijkheid van successieve antwoorden in diverse gedragssituaties. Nederlands Tijdschrift voor de Psychologie, 1964, 19, 328-340.

\section{NOTE}

1. Assume the value of $S(n-1)$ is 1 . Without knowing the previous response, this means that the expected value of $R(n)$ must be close to 1 . Now consider each of the subsequent $S(n)$ values. If $S(n)$ is $1-5, R(n+1)$ will be less than 5 , because $R(n+1)$ assimilates only part of the distance between $S(n)$ and $R(n)$. If $S(n)$ is greater than $5, R(n+1)$ will still be less than $S(n)$. Hence, the expected value of $R(n+1)$ is less than 5 . This is an assimilation effect, not a contrast effect.

(Received for publication August 26, 1980; accepted February 9, 1981.) 